The Astrophysical Journal Supplement Series, 90:743-751, 1994 February

(c) 1994. The American Astronomical Society. All rights reserved. Printed in U.S.A.

\title{
QUIESCENT MICROWAVE EMISSION FROM LATE-TYPE STARS
}

\author{
MANUEL GÜDEL \\ Joint Institute for Laboratory Astrophysics, University of Colorado and National Institute of Standards and Technology, Boulder, CO $80309-0440$ \\ Received 1993 February 26; accepted 1993 June 21
}

\begin{abstract}
A diversity of stellar classes has been detected to be prolific sources of low-level, "quiescent" microwave radiation. This emission is, in most cases, attributed to the persistent presence of mildly relativistic electrons in the coronae. Frequent or continuous particle acceleration is required to maintain a high level of gyrosynchrotron emission. In this paper, observations relevant to our understanding of quiescent microwave emission from stars are reviewed, with emphasis on nondegenerate, late-type stars. Though the nature of the acceleration process remains unknown, a large amount of crucial information is presently available, in particular observations of timescales of slow variations, estimates of particle energies, model calculations based on spectral observations, and correlative studies with soft X-ray emission.
\end{abstract}

Subject headings: acceleration of particles — radio continuum: stars - stars: activity — stars: coronae stars: late-type — stars: magnetic fields

\section{INTRODUCTION}

The presence of strong microwave emission on several classes of active, late-type stars at an apparently nonflaring, quiescent level has been challenging ever since its first discovery. Our present understanding is that this emission does not necessarily imply a steady phenomenon, but requires the persistent acceleration of electrons to high energies in the stellar coronae. This emission sharply contrasts with the orders of magnitude less intense microwave radiation of the quiet Sun, which at centimeter wavelengths comes from free-free emission at the transition-region level $\left(T \approx 2-5 \times 10^{4} \mathrm{~K}\right.$; Dulk 1985 ), and from slowly variable thermal gyroresonance emission in strong coronal magnetic fields (Kundu 1965; Dulk \& Gary 1983).

We are still learning about the processes in stellar coronae that continually supply accelerated particles. Are particles being accelerated continuously or repeatedly? Are they efficiently trapped in coronal loops after their episodic acceleration during large flares? What is the role of particle acceleration in coronal heating mechanisms?

Although we are nowhere near a conclusive answer on any of these basic questions, variability studies, modeling of energetics, spatially resolved observations, and coordinated multiwavelength investigations may provide key information for a deeper understanding. In the present summary of stellar microwave observations I will emphasize properties of nonthermal, quiescent emission that are common to different classes of late-type active stars, and that are relevant to investigations on the role of trapped, accelerated particles in stellar atmospheres. I will include chemically peculiar Bp/Ap stars despite their "early" spectral type, since they may share relevant properties with cooler stars. Many aspects of quiescent radiation have been discussed in previous reviews, of which I mention those by Dulk (1985), Bastian (1990), Kundu \& White (1992), and Drake (1993).

\section{LATE-TYPE MAIN-SEQUENCE STARS}

Late-type main-sequence dwarfs are prolific steady microwave emitters (e.g., Gary \& Linsky 1981; Fisher \& Gibson 1982; Topka \& Marsh 1982; Linsky \& Gary 1983; and others); the bulk of the detections shows a clear tendency toward emission-line M dwarfs (surveys by White et al. 1989a; Güdel et al. $1993 \mathrm{~b}$ ) as well as young objects which are subject to rapid rotation and hence the generation of a strong magnetic dynamo (see discussion in White, Jackson, \& Kundu 1993). Strong emission in the hydrogen lines is an indicator of chromospheric heating processes (e.g., Cram \& Mullan 1979); indeed, $M$ stars are also surprisingly strong coronal X-ray sources at high temperatures $\left(\sim 10^{7} \mathrm{~K}\right.$; Swank \& Johnson 1982); soft X-ray luminosities exceed the solar quiescent luminosity $\left(\approx 10^{27} \mathrm{ergs} \mathrm{s}^{-1}\right)$ by up to about three orders of magnitude (Vaiana et al. 1981).

The prospect of linking activity on the coolest stars with true solar-like activity prompted several searches for microwave emission on earlier-type stars, notably nearby dKe stars. I should mention here reports on the G star $\chi^{1}$ Ori (Gary \& Linsky 1981; though the radiation may in fact originate from a M dwarf companion, see Linsky \& Gary 1983; Pallavicini, Willson, \& Lang 1985); two possible quiescent detections of K-type BY Dra stars (Caillault, Drake, \& Florkowski 1988); the young, nearby Pleiades-age dK0e star HD 82558 (quoted in Drake et al. 1991); a few K stars selected for indicators of strong chromospheric and coronal activity (Güdel 1992); also the evolved F5 IV-V star Procyon (Drake, Simon, \& Brown 1993 ), and the conspicuously active, very young K0 Ve stars AB Dor (e.g., Lim et al. 1992) and "Speedy Mic" (Brown et al. 1994) that further suggest that strong radio emission from latetype dwarfs is often linked to an early evolutionary stage (e.g., White, Pallavicini, \& Kundu 1992a). The solar-like stars of spectral class GV are represented by at least four strong detections (Güdel, Schmitt, \& Benz 1993); one of them (HD 
129333 ) is a young, Pleiades-age rapid rotator, while another (HR 9107) is, unexpectedly, an old, metal-deficient dwarf moving off the main sequence. Such observations are, however, complicated by the possible presence of undetected cooler companions.

\subsection{Characteristics of the Radiating Particles in $M$ Dwarf Coronae}

For steady microwave sources, the concept of nonthermal gyrosynchrotron emission from accelerated $\mathrm{MeV}$ particles has become established mainly on two grounds: (1) Estimates of brightness temperatures on the reasonable assumption of a source size equal to the stellar disk clearly require a nonthermal electron population $\left(T_{B} \approx 10^{8}-\right.$ few times $10^{10} \mathrm{~K}$; White et al. 1989a). Benz \& Alef (1991) found YZ CMi at a low, though highly polarized flux in VLBI observations; unresolved emission puts $T_{B} \gtrsim 1.7 \times 10^{9} \mathrm{~K}$, clearly in the nonthermal regime. (2) Relatively flat spectra with turnover frequencies around $5 \mathrm{GHz}$ disagree with thermal models (Kundu \& Shevgaonkar 1985; Slee et al. 1988).

There is abundant evidence (e.g., starspots, and direct measurements of magnetic surface fields, see Saar \& Linsky 1985) that suggests that coronae on dwarf stars are structured into an ensemble of solar-like, localized, magnetic loops. Corresponding model calculations can provide us with more information on the energy distribution of the underlying particle population (White, Kundu, \& Jackson 1989b). For the rather typical observations of microwave spectra in Figure 1, the acceptable fits found in White et al.'s numerical gyrosynchrotron calculations are for energy power-law indices $\delta=3$ or 4 (and a surface field $B_{0}=5 \times 10^{2}$ or $1 \times 10^{3} \mathrm{G}$ ). The observed spectral index between the two highest (optically thin) frequencies predicts $\delta \approx 3.3$ for a homogeneous source (see Dulk \& Marsh 1982). Further evidence comes from a microwave dMe star survey that yields, with a wide scatter, a median of -0.3 for the $4.9-$ 8.5 GHz spectral index (Güdel et al. 1993b); this is flatter than the spectra in Figure 1 and may indicate slightly harder $(2.5 \lessgtr$ $\delta \lesssim 3$ ) energy distributions up to energies of a few MeV.

Very high-frequency observations should allow us to improve determination of $\delta$, the magnetic field, and a possible high-energy cutoff of the accelerated electron population. The few published $\mathrm{mm}$ observations may, however, require alternative explanations (e.g., Doyle \& Mathioudakis 1991; Mullan et al. 1992).

I note for completeness that while gyrosynchrotron emission from accelerated electrons is most probably the main constituent of quiescent emission, other components cannot be excluded. Thermal gyrosynchrotron emission from a tenuous $\left(10^{-3}\right.$ of ambient density) high-temperature $\left(\sim 4 \times 10^{8} \mathrm{~K}\right)$ component embedded in the cooler bulk plasma has successfully been applied to observations (e.g., Linsky \& Gary 1983; Gary 1985, 1986). Thermal gyroresonance models $\left(T \approx 10^{7}\right.$ $\mathrm{K}, \sim \mathrm{kG}$ magnetic fields) may occasionally explain $\mathrm{U}$-shaped microwave spectra (Cox \& Gibson 1985; Güdel \& Benz 1989). Coherent processes may be responsible for a few observations that exhibit very strong polarization degrees up to $90 \%$ or narrow emission bandwidth (as little as 10\%; Lang \& Willson 1986, 1988a; Benz \& Alef 1991).

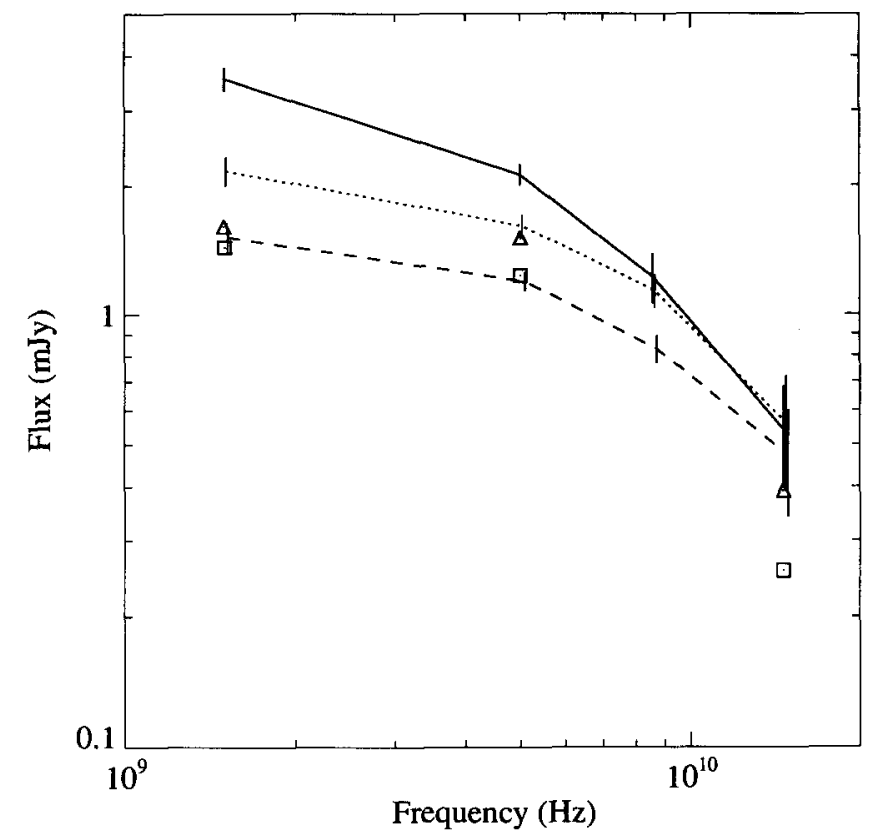

FIG. 1.-Three microwave spectra of the dMe star UV Cet, obtained on different days with the VLA at $1.4,4.9,8.4$, and $14.5 \mathrm{GHz}$ (Güdel \& Benz 1994). Note the similar shape of the spectra, while flux levels are different. The $14.5 \mathrm{GHz}$ detection of the lowest spectrum is rather marginal, and may be lower $(3 \sigma=0.39 \mathrm{mJy}$ ). The squares describe a model spectrum with energy spectral index $\delta=3$ and surface magnetic field $=500$ $\mathrm{G}$, scaled to the lowest of the observed spectra; similarly, the triangles are for a model with $\delta=4$ and a surface magnetic field of $1000 \mathrm{G}$ (models from White et al. 1989b).

\subsection{Variability Timescales and Polarization}

The notion of a steady, constant microwave emission is certainly inappropriate if we consider its typical variability over factors of $\sim 2$ on timescales of months to years (Pallavicini et al. 1985). Even larger amplitude variations have been observed (YY Gem: Linsky \& Gary 1983; UV Cet: Bastian \& Bookbinder 1987; YZ CMi: Lang \& Willson 1986); some of them may occasionally be ascribed to modulations by the stellar rotation (AU Mic: Cox \& Gibson 1985; YY Gem: Gary 1985, 1986; AB Dor: Lim et al. 1992). As illustrated in Figure 2 , short modulations occur over tens of minutes to hours (Jackson, Kundu, \& White 1989; see also Linsky \& Gary 1983; Pallavicini et al. 1985; Lang \& Willson 1986; Willson, Lang, \& Foster 1988). The shortest variations have been reported to typically exceed one minute (Kundu et al. 1988; Jackson et al. 1989), which may point to possible timescales of repeated particle acceleration or trapping times. Contrary to widespread assumption, there is no clearcut indication of continuous, short time-scale radio microflaring at sub-minute timescales as a principal constituent of quiescent emission.

Polarization measurements provide another important discriminator for both the responsible emission mechanism and the relevant particle energies. For single sources, moderate but nonzero circular polarization suggests (1) mildly relativistic electrons, (2) strong magnetic fields, (3) not too hard electron distributions, and (4) optically thin radiation (see Dulk \& Marsh 1982 for empirical expressions). Quiescent M dwarfs 


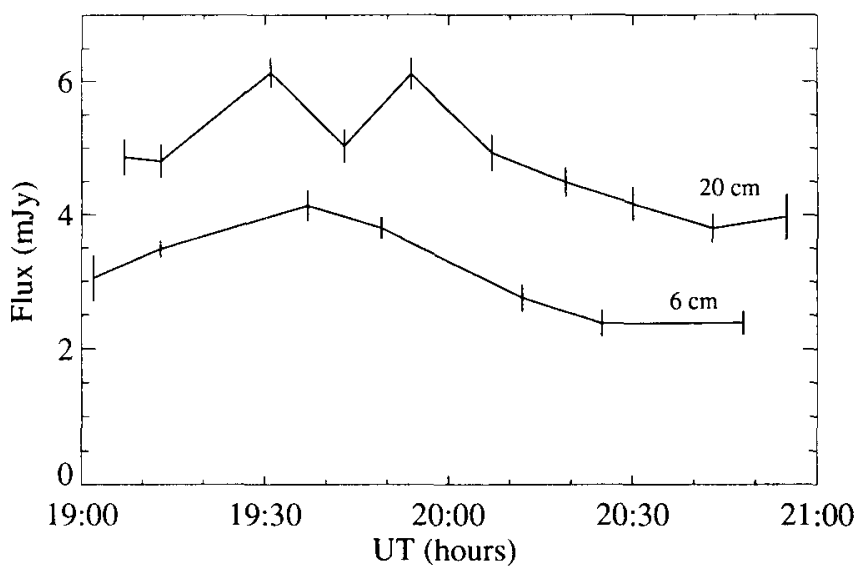

FIG. 2.-Slowly variable "quiescent" microwave emission from the $\mathrm{dMe}$ star UV Cet at wavelengths of $6 \mathrm{~cm}$ (lower curve) and $20 \mathrm{~cm}$ (upper curve). The $20 \mathrm{~cm}$ light curve is shifted by $+1 \mathrm{mJy}$ for illustration purposes. The radiation is unpolarized and gradually varies on timescales of 10-30 minutes similarly at both wavelengths. (After Jackson et al. 1989 data courtesy of S. M. White.)

usually show rather low polarization such that its absence has occasionally been considered to be a characteristic property; however, polarization degrees up to $50 \%$ are possible in quiescence (Linsky \& Gary 1983; Slee et al. 1988; Lim 1993). Low net polarization may alternatively be due to canceling polarization contributions from a variety of optically thin sources with different magnetic field orientations.

\section{ACTIVE STARS ABOVE THE MAIN SEQUENCE}

Apart from cool main-sequence objects several stellar classes are known to emit continuous, very strong nonthermal microwaves ( $14 \lesssim \log L_{R} \lesssim 18$; see, e.g., survey by Slee et al. 1987a). The relevant classes are the following:

1. RS CVn binaries were defined by Hall (1976) as close, detached binary stars with periods $1 \leqslant P_{\text {rot }} \leqslant 14$ days; the definition has been relaxed to include longer period, nonsynchronously rotating systems. They typically consist of an F-G main-sequence primary and a cooler secondary at a more evolved stage; furthermore, these stars are abnormally strong Ca II $H$ and $K$ line emitters, give evidence of large starspots, and are vigorous sources of thermal $\mathrm{X}$-rays.

2. Algol-type binaries are close, semi-detached binaries typically consisting of a B-A main-sequence primary and a G-K subgiant. The latter fills its Roche lobe and transfers mass onto the primary via a gas stream and an accretion disk. The secondary is thought to possess an active chromosphere and corona, where radio and X-rays originate.

3. FK Comae-type stars are single, rapidly rotating $\left(v \sin i \gtrsim 100 \mathrm{~km} \mathrm{~s}^{-1}\right.$ ) late-type (G-K) giants or subgiants (Bopp \& Stencel 1981 ) with intense Ca II $H$ and $K$ line emission. They are believed to have resulted from the coalescence of close (contact) binaries when one of the companions evolved into a giant.

4. Post-T Tau stars (PTTS) are young stars of low mass thought to have evolved beyond the classical T Tau phase, and moving toward the late main sequence. Many of them are sources of strong X-rays and microwaves (White et al. 1992a, and references therein ). Recently, classical $\mathrm{T}$ Tau objects, long thought to be thermal wind sources only, have also been found to support nonthermal radiation (Phillips, Lonsdale, \& Feigelson 1993; Skinner \& Brown 1994; see also discussion in André et al. 1992).

RS CVn and Algol binaries were among the first detected coronal sources of steady microwaves (Wade \& Hjellming 1972; Hjellming, Wade, \& Webster 1972). Comprehensive statistical studies do not reveal significant phenomenological differences in the microwave behavior of the two classes (e.g., Gibson 1985; Drake, Simon, \& Linsky 1986, 1989, 1992; Umana, Catalano, \& Rodonò 1991); PTTS also exhibit conspicuous similarity to RS CVn binaries in almost all microwave characteristics (Feigelson \& Montmerle 1985; Skinner 1993); even the radiation of the single giant FK Comae stars compares well with RS CVn stars (Hughes \& McLean 1987).

\subsection{Microwave Characteristics and a Simple Trap Model}

In contrast to main-sequence stars, the classes considered here seem to support a continuous spectrum of variability timescales and amplitudes steadily evolving between long periods of low, "quiescent" levels and very energetic outbursts. Typical variations of the low-level emission occur on timescales of hours or days (Spangler 1977; Mutel \& Weisberg 1978; Willson \& Lang 1987; Lang \& Willson 1988b; Massi \& Chiuderi Drago 1992; Phillips, Lonsdale, \& Feigelson 1991).

A clear distinction between flaring and "quiescent" emission is probably impossible on the basis of flux and polarization measurements alone (Lestrade et al. 1984; Mutel \& Lestrade 1985); in fact, the similar luminosity functions of RS CVn stars and PTTS, both either single exponential-like functions or very flat distributions, may indicate that a spectrum of flarelike modulations continues toward smaller amplitudes, and may cast doubt on the concept of a truly "quiescent" emission level (Owen \& Gibson 1978; Slee et al. 1987a; Mutel et al. 1987; Leous et al. 1991).

Gyrosynchrotron emission from solar-like, magnetic coronal structures is the proposed mechanism for both flaring and low-level emissions (e.g., Gibson \& Hjellming 1974; Owen, Jones, \& Gibson 1976; Spangler 1977; Mutel et al. 1987; White, Pallavicini, \& Kundu 1992b). For RS CVn binaries, Drake et al. $(1989,1992)$ argued in favor of a tenuous thermal component at $\mathrm{T} \approx 5 \times 10^{8} \mathrm{~K}$, but VLBI observations (yielding higher $T_{B}$ ) and spectral considerations (Chiuderi Drago \& Klein 1990; for PTTS: Bieging, Cohen, \& Schwartz 1984; Bieging \& Cohen 1989; Skinner 1993) now unequivocally support the nonthermal model with Lorentz factors $\gamma \leqslant 5$ for $\mathrm{cm}$ emission (Table 1). Chiuderi Drago \& Franciosini (1993) studied the dynamic evolution of the trapped particle population during "quiescence"; though collisional and synchrotron losses modify their injection distribution, they find that spectral observations are compatible with an initial power-law electron distribution, while any thermal model encounters serious diffculties with regard to the radial dependence of the magnetic field. Measurements of the complete spectral shape up into the millimeter range could provide final clarification ( for observations, see Epstein \& Briggs 1978; White et al. 1990).

The Very Long Baseline Interferometry (VLBI) technique has resolved several RS CVn and Algol systems as well as 
TABLE 1

Typical Source Parameters of Microwave Emission from ACtive Stars ANd the Sun ${ }^{a}$

\begin{tabular}{|c|c|c|c|c|c|c|}
\hline Stellar Class & $\begin{array}{c}\text { Source Size } \\
\left(10^{11} \mathrm{~cm}\right)\end{array}$ & $\begin{array}{c}\log T_{B} \\
(\mathrm{~K})\end{array}$ & $\begin{array}{c}B \\
(\mathrm{G})\end{array}$ & $\begin{array}{l}\gamma \text { for } \mathrm{cm} \\
\text { Radiation }\end{array}$ & $\delta$ & Timescales \\
\hline $\mathrm{dM} / \mathrm{dK} / \mathrm{dG}$, quiescent & $\lesssim 1$ & $9 \pm 1$ & $\sim 100$ & $(3-7)$ & $2.5-3$ & minutes-hr \\
\hline RS CVn, quiet halo ......... & $10-30$ & $9 \pm 0.6$ & $5-50$ & $2-3.5$ & $2-4$ & days \\
\hline Algols, core $\ldots \ldots \ldots \ldots \ldots$ & $0.7-2$ & $9.5 \pm 0.5$ & $40-80$ & $4-7$ & $2-3$ & $\mathrm{hr}$ \\
\hline Algols, burst & $2-8$ & $10.3 \pm 0.2$ & $4-15$ & $4-7$ & $2-3$ & $\mathrm{hr}$ \\
\hline Algols, quiet halo & $2-8$ & $9.1 \pm 0.6$ & $10-20$ & $\leqslant 3-5$ & $2-4$ & days \\
\hline PTTS, halo . . . . . . . . . & $10-100$ & $8.2 \pm 0.6$ & $0.1-100$ & $(\gtrsim 3)-5$ & $\ldots$ & hr-days \\
\hline $\mathrm{Bp} / \mathrm{Ap}$, quiescent $\ldots \ldots \ldots$ & $10-30$ & $9.8 \pm 0.3$ & $60-160$ & (4-9) & $2-3$ & $\mathrm{hr}$ \\
\hline Solar gradual flare $\ldots \ldots \ldots \ldots$ & $0.01-0.1$ & $(8-9 ?)$ & $\lesssim 100$ & $\gtrsim 3$ & $2-3.5$ & $\sim 1 \mathrm{hr}$ \\
\hline Solar impulsive flare...$\ldots$. & $\sim 0.01$ & $7-9$ & few 100 & $\$ 1.5$ & variable & $\sim 1$ minute \\
\hline
\end{tabular}

a Principal references used: dMe-White et al. 1989a, b, Benz \& Alef 1991; RS CVn/Algols—Owen et al. 1976, Owen \& Spangler 1977, Spangler 1977, Doiron \& Mutel 1984, Mutel et al. 1984, 1985, Lestrade et al. 1984, 1985, 1986, 1988, Lestrade 1988, Kuijpers \& van der Hulst 1985, van den Oord et al 1989, Morris et al. 1990, Massi \& Chiuderi Drago 1992, Chiuderi Drago \& Franciosini 1993, Umana et al. 1993; FK Comae--Hughes \& McLean 1987, Slee et al. 1987b; PTTS-Phillips et al. 1991, White et al. 1992a, Skinner 1993; Bp/Ap-Drake et al. 1987, Phillips \& Lestrade 1988, Linsky et al. 1992; Sun-Dulk 1985, Cliver et al. 1986, Dennis 1988, D. Gary, private communication. Values in parentheses are estimates derived from literature values for $\sim 5 \mathrm{GHz}$

PTTS and provided conclusive evidence for a core + halo structure during various episodes (Mutel et al. 1985; Phillips et al. 1991). The very extended quiescent RS CVn "halo" with a size comparable to the binary system has been modeled as a dipole-like magnetosphere containing trapped, accelerated particles in an equatorial belt ("dead zone model"; Morris, Mutel, \& Su 1990; similar to Fig. 3 below); the model seems to provide a correct framework for PTTS as well (Phillips et al. 1991; Skinner 1993). It predicts energy power-law indices $\delta \approx$ 3.5 during quiescence.

In an attempt to explain the dynamics of the observed emission characteristics of RS CVn stars, Mutel et al. (1985) proposed a three-step model scenario that links all activity stages; similar models seem to apply to Algols (Lestrade et al. 1988): (1) During an outburst in compact coronal loops with $B \approx$ $100 \mathrm{G}$ in the low corona of the active component, the VLBI "core," particles are accelerated to at least $1-5 \mathrm{MeV}$ (relevant for $6 \mathrm{~cm}$ emission); they give rise to optically thick, unpolarized, nonthermal gyrosynchrotron emission. (An alternative model by Uchida \& Sakurai 1983, which relies on intrabinary reconnection regions may now be questioned, since the microwave activity is strongly related to the "active" single star, or stars, in the binary system; see Morris \& Mutel 1988.) (2) As the coronal loops expand on timescales of 10-30 hours ("flare decay"), the plasma becomes increasingly optically thin to gyrosynchrotron emission; therefore, the microwave spectral index flattens (decreases), and the polarization increases as the flux decreases (e.g., Mutel et al. 1987). (3) The "quiescent" phase is identified with the flare "remnant," when magnetic loops have expanded to the size of the binary system; fully optically thin gyrosynchrotron emission from $\mathrm{MeV}$ electrons trapped in low intrabinary magnetic fields (of order of $10 \mathrm{G}$; the VLBI "halo") evolves on timescales of several days. Spectral indices within $-0.9 \leqslant \alpha \leqslant 0.1, T_{B} \approx a$ few times $10^{8} \mathrm{~K}$ (Mutel et al. 1987; also Bieging et al. 1984, Bieging \& Cohen 1989, and Skinner 1993 for PTTS) and mild circular polariza- tion prevail. Circular polarization during quiescence has been a strong argument in favor of the gyrosynchrotron mechanism (Owen et al. 1976; Spangler 1977). Fractional polarization degrees as high as $40 \%-50 \%$ have occasionally been observed (Feldman et al. 1978; Gibson, Hicks, \& Owen 1978) though they range typically below 20\% (Weiler et al. 1978; Drake et al. 1992) and may often not exceed 5\% (Spangler 1977; Doiron \& Mutel 1984). In the only marked difference, PTTS often show very weak or no measurable polarization during phases of no obvious flaring (Phillips et al. 1991; White et al. 1992b; Skinner 1993 gives $19 \%$ for Hubble 4 ).

Hughes \& McLean (1987) model the FK Comae microwave source in terms of gyrosynchrotron emission from coronal streamers; they arrive at a very hard electron energy power-law index of $\delta \approx 2$, with $B \approx 15 \mathrm{G}$. An extremely high percentage of about $20 \%$ of the particles may become accelerated into the nonthermal tail above $10 \mathrm{keV}$ (see also Mullan 1985 for similar results on RS CVn outbursts).

\section{CHEMICALLY PECULIAR B AND A STARS}

Moving toward earlier spectral types on the main-sequence, we expect that convective layers in the stellar interior (responsible for a magnetic dynamo) become negligible at spectral type A. Nevertheless, it is well-known that stars of chemically peculiar spectral types $A p$ and $B p$ possess surface magnetic fields of kG strength (Babcock 1947; see Borra, Landstreet, \& Mestel 1982), thought to be organized in a global, dipolar structure.

Drake et al. (1987) and Linsky, Drake, \& Bastian (1992) report microwave detections with a strong tendency to the earlier spectral-type (B) subclasses, with radio luminosities in the range of $\mathrm{RS} C V n$ binaries, i.e., $14.5 \leqq \log L_{R} \lesssim 17.9$. Analogous to RS CVn binaries, circular polarization tends to be low to moderate $(\leqslant 20 \%)$, variability may occur on timescales of a few hours, and the microwave spectra are generally flat, with a peak flux around $5 \mathrm{GHz}$. Brightness temperatures greater than 
$10^{9} \mathrm{~K}$ ( based on VLBI observations; Phillips \& Lestrade 1988) support, like on other active stars, a nonthermal gyrosynchrotron process. Interestingly, however, observations show that $L_{6 \mathrm{~cm}} \propto \dot{M}^{1 / 2} B_{\text {rms }}$, where $\dot{M}$ is the mass loss rate by winds (Linsky et al. 1992). That radio emission is dependent both on mass loss and the magnetic field may be understood in terms of a magnetospheric model (Fig. 3) proposed by Havnes \& Goertz (1984) and subsequently refined and applied to Ap/Bp stars by Linsky et al. In this model, most of the mass outflow occurs at high stellar latitudes, where the dipole-shaped magnetic fields become nearly radial. At some radius $(\sim 10-$ $20 R_{*}$ ), the thermal wind pressure exceeds the magnetic pressure, drawing the magnetosphere into a current sheet configuration in the equatorial plane similar to planetary magnetotails. The current sheets are assumed to be the sources of "coronal" heating and of inward accelerated particles that subsequently become trapped. Nonthermal energy power-law indices of $\delta \approx 2-3$ reasonably agree with the observations. Rotational modulation observed by Leone \& Umana (1993) supports the general characteristics of the model. I note in passing that a similar model has been invoked for a very young magnetic B star (André et al. 1988).

\section{CHARACTERISTICS OF STELLAR GYROSYNCHROTRON SOURCES}

It is interesting in itself that very different single or binary stars, young or evolved systems support magnetic energy conversion mechanisms that apparently lead to similar microwave characteristics. To support this view, I compile in Table 1 some gross characteristics of several stellar (and solar) microwave sources, approximately representing and comprising the bulk of observational data and model estimates given in the literature. Such a compilation is necessarily incomplete, in part inaccurate and at places too restrictive due to variable coronal conditions, simplistic, differing, and biased model assumptions, or too few representative observations; it nevertheless gives some overall indications from many independent sources and methods.

A few related stellar classes that might show similar nonthermal phenomena have not been included here; I mention observations of early-type pre-main-sequence "Herbig" A and B stars (usually thermal sources; see Skinner, Brown, \& Stewart 1993), Hybrid stars (possessing winds and coronae; see Drake \& Linsky 1986), W UMa contact binaries (very few detec-

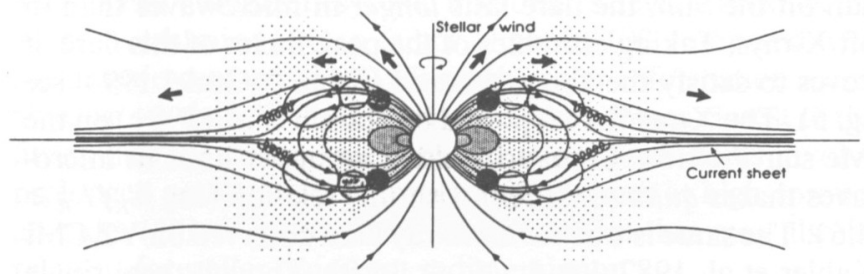

FIG. 3.-Cross section through a model of a Bp star magnetosphere. A wind originates from high stellar latitudes; at some distance, it draws the approximately dipolar magnetic field lines into a current sheet configuration where plasma heating and particle acceleration occur. Accelerated particles travel along the lines and are trapped in the inner zone while radiating gyrosynchrotron emission. (From Linsky et al. 1992.) tions; see Hughes \& McLean 1984), and a few nonthermal O-star sources (usually dominated by winds, very low $B$; see Bieging, Abbott, \& Churchwell 1989).

\section{MICROWAVES AND THERMAL SOFT X-RAY RADIATION}

\subsection{Information from Soft $X$-Rays on the Energy Release Processes}

The persistent presence of accelerated electron populations in an apparently quiet, nonflaring stellar corona is puzzling, especially in view of various energy loss mechanisms (collisions, radiation, etc). Unfortunately, hard X-rays, the primary indicators of accelerated particles, cannot routinely be monitored on stars due to sensitivity limits of present-day instruments. Microwaves are very sensitive diagnostics for nonthermal particles, but they are unimportant energetically. Soft $\mathrm{X}$-rays, on the other hand, have become the crucial tool to study coronal energy release processes, investigate radiative and conductive losses, and derive temperatures and emission measures of the emitting plasma. For stellar coronal sources, this radiation is assumed to be fully thermal ( see discussion in Drake et al. 1992).

The quiescent soft X-ray flux on dMe stars was found to be proportional to the time-averaged flare energy in the Johnson $U$-band (Doyle \& Butler 1985; Skumanich 1985; Whitehouse 1985). It may either imply that flaring energy and coronal heating share a common origin, or that flares themselves deposit enough heat into the corona, possibly an ensemble of numerous small-scale flares, or single large flares combined with a large coronal heat capacity.

Heating by numerous small-scale "microflares" was suggested for the Sun (Lin et al. 1984), based on hard X-ray observations and their $N(S)$ flux (or energy) distribution; the total energy deposited in the chromosphere by precipitating accelerated particles could be sufficient to maintain the thermal energy level in the corona. Precipitation and thus nonthermal energy deposition in the chromosphere may be even more important on dMe stars due to their high plasma temperatures combined with their surface gravity (Kundu et al. 1987). Monitoring of flare stars in X-rays in fact seems to reveal copious small, flarelike variations that correlate with chromospheric $\mathrm{H} \gamma$ line emission (Butler et al. 1986), though this does not necessarily imply heating by precipitating electrons. It is also interesting that these stars seem to consistently support an X-ray emitting, high-temperature plasma component (Swank \& Johnson 1982; Schmitt et al. 1987, 1990); could this component be a flare plasma that is continuously heated by smallscale, flarelike events?

Ambruster, Sciortino, \& Golub (1987) searched for microvariability in the steady X-ray emission of a large sample of dMe star X-ray observations by Einstein. They concluded that almost all bright light curves vary significantly up to about $30 \%$ on timescales of several hundreds of seconds, but the $N(S)$ distribution of these events does not support a connection between low-level flaring and coronal heating. This result can not, however, be conclusive since the weakest detectable X-ray flares are still comparable to strong solar flares, and the presence of low-level flaring below the detection threshold cannot be excluded. In a similar study, EXOSAT data did not reveal short time-scale fluctuations above the detection limit, but did 
show gradual variations over minutes to hours (Pallavicini, Tagliaferri, \& Stella 1990; Collura, Pasquini, \& Schmitt 1988).

\subsection{A Relation between Coronal $X$-Ray and Radio Emission on Stars}

The importance of X-ray observations with regard to energy release processes and the diagnostic potential of microwaves to probe accelerated particles motivated several correlative studies. Bookbinder ( 1987) reports a dependence between the nonsimultaneously measured radio and X-ray luminosities $L_{R}$ and $L_{\mathrm{X}}$ of dMe stars (see also Katsova 1987). The existence of such a relation was questioned by Caillault et al. (1988) and Caillault (1989), who considered sensitivity limits, long-term variations, and the different origins of the emissions to explain what they saw as an absence of correlation. On the other hand, radio-detected dMe stars of a given spectral type tend to be those with the highest $\mathrm{X}$-ray fluxes, which at least indicates that the overall coronal activity reflects similarly both in the X-ray and the microwave losses (White et al. 1989a). For the RS CVn class, Drake et al. ( 1986, 1989, 1992) report a distinct relation between $L_{R}$ and $L_{\mathrm{X}}$ close to proportionality: $\log \left(L_{\mathrm{X}} / L_{\mathrm{bol}}\right)$ $=(1.16 \pm 0.12) \log \left(L_{R} / L_{\mathrm{bol}}\right)+$ constant.

Correlation of nonsimultaneous observations of $L_{\mathrm{X}}$ and $L_{R}$ is handicapped by slow variations in both emissions $(\$ \S 2.2$, $3.1,4)$. Kundu et al. (1988) obtained exactly simultaneous monitoring of four radio- and X-ray-bright dMe flare stars. In this sample, quiescent $L_{\mathrm{X}}$ and $L_{R}$ were not correlated. It seems, however, that this result was influenced by the selection of the "prototype" flare star UV Cet and two other stars at similar $L_{R}$ (see below).

A recent program took advantage of the all-sky survey obtained by ROSAT (Güdel et al. 1993a, b). The VLA obtained mostly simultaneous observations at $20 \mathrm{~cm}, 6 \mathrm{~cm}$, and $3.6 \mathrm{~cm}$ for $19 \mathrm{dMe}$ target systems (including visual and spectroscopic binaries). The optically thin $6-3.6 \mathrm{~cm}$ portions of the spectra were correlated in luminosity with the $0.1-2.4 \mathrm{keV}$ X-rays. This sample clearly indicates a strong correlation suggesting proportionality: $\log L_{\mathrm{X}} \approx(0.99 \pm 0.11) \log L_{R}+(15.57 \pm$ 1.43) with a high correlation coefficient of $R=0.90$ for 14 detections. The correlation tightens once the analysis is confined to strictly simultaneous time intervals, yielding $R=0.95$ for nine detections. The prototype flare star UV Cet $(\equiv \mathrm{G} 1$ $65 \mathrm{~B}$ ) is recognized as a microwave-rich exception (Figs. 4 and 5). In a similar ROSAT and VLA study of $15 \mathrm{RSCVn}$ binaries, Fox et al. ( 1993) find $\log L_{\mathrm{X}} \approx(1.04 \pm 0.26) \log L_{R}+14.2$ for simultaneous observations.

It is tempting to extend such correlations to include different classes of active coronal objects. Figure 4 (based on the available literature; see Güdel \& Benz 1993) suggests that the separate trends on RS CVn binaries and dMe stars pertain to the same overall relation that includes other active stellar classes. Since radio and $\mathrm{X}$-rays were not simultaneously measured in the upper right quadrant, much scatter is introduced by the inherently large variability of the low-level radio emission. The overall relation pertains to coronally active stars and is, for these objects, independent of spectral type, stellar age and evolutionary state, binarity, rotation period (between 12 hours and $\sim 100$ days), or photospheric/chromospheric activity (Güdel \& Benz 1993). It was explicitly shown that some lumi-

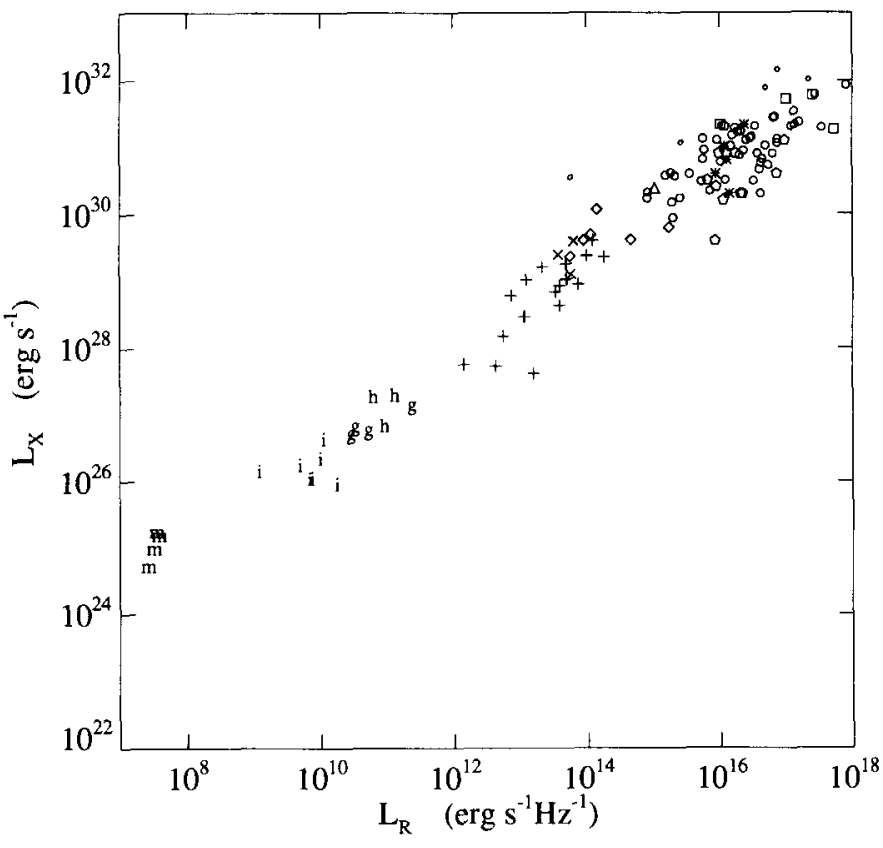

Fig. 4.-Comparison of soft $\mathrm{X}$-ray and microwave $(\approx 5 \mathrm{GHz})$ luminosities of several classes of stars ( symbols) and solar flares (letters $\mathrm{g}, \mathrm{h}, \mathrm{i}, \mathrm{m}$ ). For the latter, mean values over twice the FWHM duration in X-rays are shown. Key to the symbols: (plus signs) $\mathrm{dM}(\mathrm{e})$ stars; (crosses) $\mathrm{dK}(\mathrm{e})$ stars; (diamonds) BY Dra binaries; (large circles) RS CVn binaries; (small circles) RS CVn binaries with two giants; (triangle) AB Dor; (asterisks) Algol binaries; (squares) FK Com stars; (pentagons) PTTS. Key to the letters: (m) solar microflares; (i) intermediate impulsive solar flares; (h) gradual solar flares with dominating large impulsive phase; (g) pure gradual solar flares. (Details and all references used for this compilation have been given in Güdel \& Benz 1993 and Benz \& Güdel 1993.)

nosity scaling with distance (due to detection limitations) can not induce the correlation for RS CVn binaries (Drake et al. 1989 ) or for dMe stars (Güdel et al. 1993b). Only the status of the chemically peculiar A- and B-type stars is presently somewhat unclear in this respect; many of them seem to be deficient in X-rays (Drake et al. 1994).

\subsection{Comparison with Flares}

Though (incoherent) gyrosynchrotron flares seem to occur rarely at observable levels on dMe stars, Kundu et al. (1988) report one radio and $\mathrm{X}$-ray flare of this kind on EQ Peg. It was essentially unpolarized and showed very long, gradual light curves; $T_{B}$ suggested a gyrosynchrotron mechanism. Other than on the Sun, the flare lasts longer in microwaves than in soft X-rays. Taking estimates of the peak fluxes of this flare, it proves to satisfy the above relation (Benz \& Güdel 1994; see Fig. 5). The X-ray flare reported by Haisch et al. (1981) on the dMe star Proxima Centauri yielded an upper limit in microwaves that is quite consistent with this relation $\left(\log L_{\mathrm{X}} / L_{R} \gtrsim\right.$ 14.6). The same is true for an X-ray flare observed on YZCMi (Kahler et al. 1982; $\log L_{\mathrm{X}} / L_{R} \gtrsim 14.0$ ). Though these single cases with regard to dMe stars that I am aware of do not provide a solid argument, it may seem suggestive that quiescent radiation is closely related to or based on similar mechanisms as flaring processes. A possible counter-example is provided for low-level, "nonflare" variations on the RS CVn system TY 


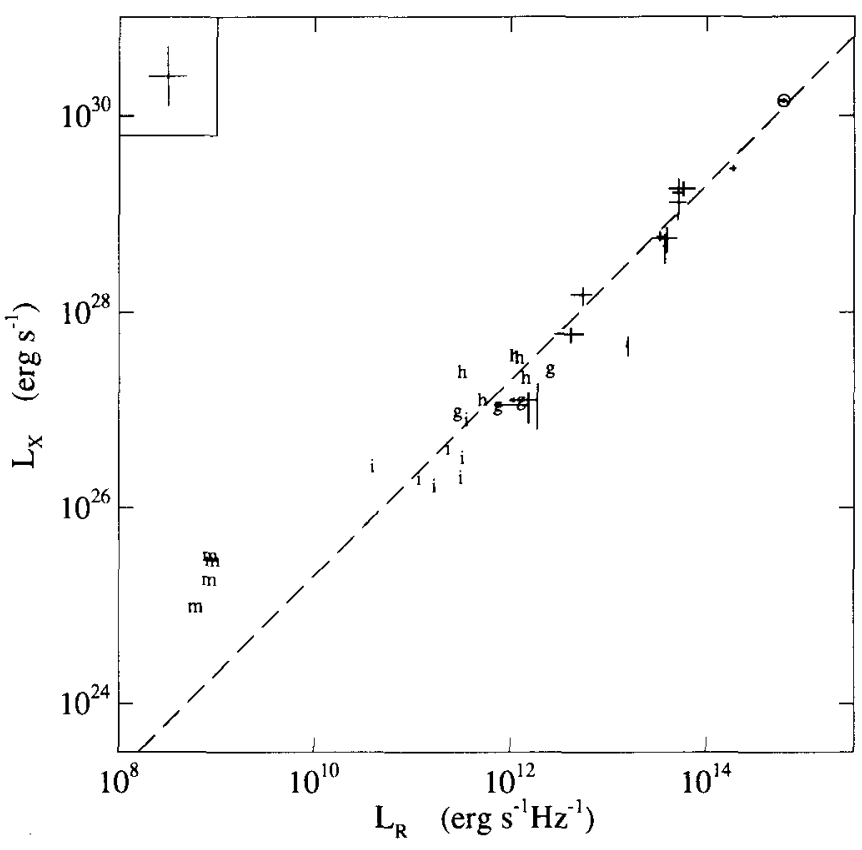

FIG. 5.-Correlation of simultaneously observed soft X-ray and microwave emissions from quiescent dMe stars and solar flares (peak luminosities have been used for the flares). The dMe stars are represented with error bars (Güdel et al. 1993b), the letters refer to the solar flares (for the key see Fig. 4). The cross at top left defines estimated mean errors for the solar flares. A long duration flare on the M dwarf EQ Peg B is shown by $\odot$. Its microwave emission has been interpreted by the gyrosynchrotron mechanism (Kundu et al. 1988). The solid line is a best-fit to the quiescent dMe stars with slope 1 (linear relation). The object significantly to the right of the fitting line is the UV Cet system. (From Benz \& Güdel 1994.)

Pyx; an increase of the microwaves by a factor of 2 went unnoticed in the X-rays (Fox et al. 1993).

The Sun supports considerable nonthermal gyrosynchrotron emission only during flaring episodes when electrons are accelerated up to at least several MeV. For peak flare fluxes, a proportionality $L_{R} \propto L_{\mathrm{X}}$ seems to apply (Drake et al. 1989). An investigation on a sample of solar gradual ("type C") and impulsive ("type B") (see Dennis 1988, and references therein) as well as "micro-" flares indeed reveals that the average flare luminosities define the gross continuation of the quiescent stellar relation, though the overall trend differs from proportionality (slope $0.73 \pm 0.03$; see Fig. 4 ; Benz \& Güdel 1994). A few solar flares fulfill the stellar relation quite well, though many of them are somewhat "X-ray-rich" (Fig. 4; Fig. 5 with peak luminosities). The microflares deviate most, being $\mathrm{X}$-ray overluminous by a factor of about 100 compared with stellar quiescent emission. Using the solar information available, we see that the class of gradual flares comes closest to the fitting line for stellar quiescent radiation. Such flares involve prolonged acceleration or long trapping times at high coronal altitudes (Cliver et al. 1986), a requirement that may also be relevant for stellar quiescent radio emission.

To round off this speculation, I would like to point out a few striking, though purely phenomenological analogies that may deserve further investigation (see Table 1). Stellar quiescent microwave radiation is conspicuously similar to the gradual (as opposed to impulsive) type of solar flares for a number of reasons. (1) Gradual solar flares usually evolve to a relatively hard photon energy index around $3 \leqslant \gamma_{p} \leqslant 3.5$ (Dennis 1988), about in accordance with $\delta$ for stellar quiescent emission (Table 1). (2) The microwave peak of solar gradual flares is usually low ( $\$ 1-3 \mathrm{GHz})$, in contrast to impulsive bursts $(\gtrsim 10$ $\mathrm{GHz}$; Cliver et al. 1986); this is related to great coronal heights of the gradual flare radio sources, where $B$ and $n$ both are low; similarly, stellar quiescent emission often peaks below $5 \mathrm{GHz}$ (e.g., Fig. 1). (3) The timescales of gradual flares are relatively long (several minutes to hours), comparable to slow variations in the quiescent stellar microwave radiation. (4) The thermal plasma of gradual solar flares attains comparatively mild peak temperatures (e.g., $\approx 17 \times 10^{6} \mathrm{~K}$ in the strong burst in Benz \& Güdel 1994), while strong impulsive flares more typically reach peak temperatures at $20-40 \times 10^{6} \mathrm{~K}$ during a short time. Stellar X-ray coronae are often described in terms of two components at different temperatures, the lower temperature at about $2.5 \times 10^{6} \mathrm{~K}$, reminiscent of the quiet solar corona, and the higher temperature at about $16 \times 10^{6} \mathrm{~K}$ (Schmitt et al. 1990; Dempsey et al. 1993, with a reanalysis of Einstein SSS data). (5) The remarkable similarity in the $L_{\mathrm{X}}$ versus $L_{R}$ relation of quiescent stellar radio emission, a long-term incoherent stellar flare, and long-term solar gradual flares remains to be investigated in this context.

\subsection{Possible Implications on the Acceleration Process and Coronal Heating}

The large range of optically thin $L_{\mathrm{X}}$ and $L_{R}$ is certainly related to the volumes of the emitting sources, if one assumes similar thermal and nonthermal energy densities in all coronae (Table 1). The flux ratio of $\sim 10^{15.5} \mathrm{~Hz}$, however, seems to be a general property of active stellar coronae. Assuming a powerlaw distribution of the electron energy with energy power-law index $\delta$, the microwave emissivity can be expressed in terms of the magnetic field strength $B$, the aspect angle $\theta, \delta$, and the number density $n_{h}$ above a threshold energy $\epsilon_{0} \equiv 10 \mathrm{keV}$ (Dulk \& Marsh 1982).

Let $a$ designate the fraction of the total energy release going into accelerated particles (above $\epsilon_{0}$ ), and let $b$ designate the portion of the total energy ultimately radiated into the soft $\mathrm{X}$-ray band; for solar flares, $a / b$ is being debated, but may be of the order of unity (Dennis 1988; Withbroe \& Noyes 1977). Güdel \& Benz (1993) derive a simple relation between $L_{X}$ and $L_{R}$, which for characteristic observational parameters $(\delta \approx 3$, $\nu=5 \mathrm{GHz}$, and $\theta=30^{\circ}$ ) yields

$$
\begin{aligned}
B^{2.48} & \frac{a}{b} \tau_{0}(\alpha+1) \\
& \approx \begin{cases}3.3 \times 10^{6} & \mathrm{dMe}, \mathrm{dKe}, \mathrm{BY} \text { Dra } \\
2 \times 10^{7} & \text { RS CVn, Algols, PTTS, FK Com } .\end{cases}
\end{aligned}
$$

Here, $\tau$ is the particle lifetime, defined by its value $\tau_{0}$ at the threshold energy $\epsilon_{0} \equiv 10 \mathrm{keV}$, and its energy power-law index $\alpha$. Assuming constant $\tau$ for the high energy electrons, we see that this relation readily reproduces typical timescales from $\mathrm{dMe}$ stars (of order of 1 minute for $B \approx 100 \mathrm{G}$ ) to RS CVn binaries (days, with $B \approx 10 \mathrm{G}$; Table 1 ).

Two possible scenarios then can explain the linear relation 
between $L_{\mathrm{X}}$ and $L_{R}:$ (1) A large fraction of the energy release is channeled into accelerated particles. The high-energy $(\sim \mathrm{MeV})$, high pitch angle portion emits most of its energy in gyrosynchrotron radiation, while the bulk of the kinetic energy is in the slower (but nonthermal) electrons; these subsequently heat the ambient plasma by collisions. Heating by accelerated particles has been suggested by a number of observations of solar flares (see Dennis 1988) and stellar X-ray and chromospheric line emission (see Butler, Rodonò, \& Foing 1988). Such scenarios require highly efficient accelerators; it is unknown whether these are realized in stellar coronae. (2) Particle acceleration could be governed by the same process that heats the plasma. In the current dissipation model by Holman (1986), for example, joule heating will be balanced by radiative losses, for which observable $\mathrm{X}$-rays provide a constraint. In parallel, thermal electrons exceeding the critical velocity related to the electric field will be freely accelerated into the nonthermal tail. The observable radio emission yields the constraints for the rate of particle acceleration. Holman's model requires about 10-100 current sheets to explain the observed flux ratio. (For a similar approach to RS CVn outbursts, see Mullan 1985.) Scenarios of this kind strongly support a coronal heating mechanism of a magnetic nature. They require that the ratio $a / b$ attains a characteristic value in most active coronae.

\section{SUMMARY}

A converging picture for the "quiescent" radiation involves mildly relativistic ( $\sim \mathrm{MeV})$ electrons with a power-law distribution in energy ( $\delta \sim 3$ ) supplied by continuous or frequent acceleration events. Outstanding problems that should be addressed with present-day observing techniques include: (1) Establishing the mechanism responsible for continuous or fre- quent acceleration of particles to $\mathrm{MeV}$ energies; (2) existence and determination of an upper threshold for particle acceleration in the quiescent phase; (3) derivation of the exact (variable?) energy distribution of the accelerated electrons up to several $\mathrm{MeV}$ from microwave spectra; (4) determination of the role of small-scale flares in quiescent emission, and their contribution to the coronal energy budget; (5) efficiency of the acceleration process and (6) its coupling to heating mechanisms; and (7) the role of efficient particle traps or quasi-continuous particle acceleration in variations of the quiescent emission.

While the data presently available do not provide conclusive answers, they do suggest that the persistent presence of relativistic particles at $\mathrm{MeV}$ energies is common to most or all active stellar coronae. The energy release mechanisms at work have yet to be established; comparison with soft $X$-ray losses may suggest that although there may be additional coronal heating mechanisms at work (e.g., in the quiet Sun), particle acceleration arises along with the principal heating process in the active coronae considered here; the observations suggest that these processes are (1) magnetic in origin, (2) flarelike in character, and (3) that they appear to necessarily imply both coronal heating to $\sim 10^{7} \mathrm{~K}$ and particle acceleration to relativistic energies.

I thank A. O. Benz for many discussions on the subject of particle acceleration, J. L. Linsky for comments on the manuscript, and the referee for thoughtful comments and suggestions. This work has been supported by the Swiss National Science Foundation (grant 8220-033360) and by NASA through interagency transfer W-17,772 to the National Institute of Standards and Technology.

\section{REFERENCES}

Ambruster, C. W., Sciortino, S., \& Golub, L. 1987, ApJS, 65, 273

André, P., Deeney, B. D., Phillips, R. B., \& Lestrade, J.-F. 1992, ApJ, 401, 667

André, P., Montmerle, T., Feigelson, E. D., Stine, P. C., \& Klein, K.-L. 1988, ApJ, 335, 940

Babcock, H. W. 1947, ApJ, 105, 105

Bastian, T. S. 1990, Solar Phys., 130, 265

Bastian, T. S., \& Bookbinder, J. A. 1987, Nature, 326, 678

Benz, A. O., \& Alef, W. 1991, A\&A, 252, L19

Benz, A. O., \& Güdel, M. 1994, A\&A, in press

Bieging, J. H., Abbott, D. C., \& Churchwell, E. B. 1989, ApJ, 340, 518

Bieging, H. H., \& Cohen, M. 1989, AJ, 98, 1686

Bieging, J. H., Cohen. M., \& Schwartz, P. R. 1984, ApJ, 282, 699

Bookbinder, J. A. 1987, in Activity in Cool Star Envelopes, ed. O. Havnes et al. (Dordrecht: Kluwer), 257

Bopp, B. W., \& Stencel, R. E. 1981, ApJ, 247, L131

Borra, E. F., Landstreet, J. D., \& Mestel, L. 1982, ARA\&A, 20, 191

Brown, A., Bromage, G., Ambruster, C. W., \& Jeffries, R. D. 1994, ApJ, submitted

Butler, C. J., Rodonò, M., \& Foing, B. H. 1988, A\&A, 206, Ll

Butler, C. J., Rodonò, M., Foing, B. H., \& Haisch, B. M. 1986, Nature, 231,679

Caillault, J.-P. 1989, AJ, 97, 163

Caillault, J.-P., Drake, S., \& Florkowski, D. 1988, AJ, 95, 887

Chiuderi Drago, F., \& Franciosini, E. 1993, ApJ, 410, 301

Chiuderi Drago, F., \& Klein, K.-L. 1990, Ap\&SS, 170, 81

Cliver, E. W., Dennis, B. R., Kiplinger, A. L., Kane, S. R., Neidig, D. F., Sheeley, N. R., Jr., \& Koomen, M. J. 1986, ApJ, 305, 920

Collura, A., Pasquini, L., \& Schmitt, J. H. M. M. 1988, A\&A, 205, 197
Cox, J. J., \& Gibson, D. M. 1985, in Radio Stars, ed. R. M. Hjellming \& D. M. Gibson (Dordrecht: Reidel), 233

Cram, L. E., \& Mullan, D. J. 1979, ApJ, 234, 579

Dempsey, R. C., Linsky, J. L., Schmitt, J. H. M. M., \& Fleming, T. A. 1993, ApJ, 413, 333

Dennis, B. R. 1988, Solar Phys., 118, 49

Doiron, D. J., \& Mutel, R. L. 1984, AJ, 89, 430

Doyle, J. G., \& Butler, C. J. 1985, Nature, 313, 378

Doyle, J. G., \& Mathioudakis, M. 1991, A\&A, 241, L41

Drake, S. A. 1993, in Physics of Solar and Stellar Coronae: G. S. Vaiana Memorial Symposium, ed. J. L. Linsky \& S. Serio (Dordrecht: Kluwer), 393

Drake, S. A., Abbott, D. C., Bastian, T. S., Bieging, J. H., Churchwell, E., Dulk, G., \& Linsky, J. L. 1987, ApJ, 322, 902

Drake, S. A., \& Linsky, J. L. 1986, AJ, 91, 602

Drake, S. A., Linsky, J. L., Judge, P. G., \& Elitzur, M. 1991, AJ, 101, 230

Drake, S. A., Simon, T., \& Brown, A. 1993, ApJ, 406, 247

Drake, S. A., Simon, T., \& Linsky, J. L. 1986, AJ, 91, 1229

1989, ApJS, 71, 905

1992 ApJS, 82, 311

Drake, S. A., Linsky, J. L., Schmitt, J. H. M. M., \& Rosso, C. 1994, ApJ, in press

Dulk, G. A. 1985, ARA\&A, 23, 169

Dulk, G. A., \& Gary, D. E. 1983, A\&A, 124, 103

Dulk, G. A., \& Marsh, K. A. 1982, ApJ, 259, 350

Epstein, E. E., \& Briggs, F. H. 1978, AJ, 83, 1487

Feigelson, E. D., \& Montmerle, T. 1985, ApJ, 289, L19

Feldman, P. A., Taylor, A. R., Gregory, P. C., Seaquist, E. R., Balonek, T. J., \& Cohen, N. L. 1978, AJ, 83, 1471 
Fisher, P. L., \& Gibson, D. M. 1982, Smithsonian Astrophys. Obs. Spec. Rep. No. 392, 109

Fox, D. C., Linsky, J. L., Vealé, A., Dempsey, R. C., \& Brown, A. 1993, A\&A, submitted

Gary, D. E. 1985 , in Radio Stars, ed. R. M. Hjellming \& D. M. Gibson (Dordrecht: Reidel), 185

. 1986, in Cool Stars, Stellar Systems, and the Sun, ed. M. Zeilik \& D. M. Gibson (Berlin: Springer), 235

Gary, D. E., \& Linsky, J. L. 1981, ApJ, 250, 284

Gibson, D. M. 1985, in Radio Stars, ed. R. M. Hjellming \& D. M. Gibson (Dordrecht: Reidel), 213

Gibson, D. M., Hicks, P. D., \& Owen, F. N. 1978, AJ, 83, 1495

Gibson, D. M., \& Hjellming, R. M. 1974, PASP, 86, 652

Güdel, M. 1992, A\&A, 264, L31

Güdel, M., \& Benz, A. O. 1989, A\&A, 211, L5 1993, ApJ, 405, L63 1994 , in preparation

Guidel, M., Bookbinder, J. A., Schmitt, J. H. M. M., \& Fleming, T. 1993a, in Physics of Solar and Stellar Coronae: G. S. Vaiana Memorial Symposium, ed. J. L. Linsky \& S. Serio (Dordrecht: Kluwer), 383

Güdel, M., Schmitt, J. H. M. M., \& Benz, A. O. 1993, BAAS, 25, 874

Güdel, M., Schmitt, J. H. M. M., Bookbinder, J. A., \& Fleming, T. 1993b, ApJ, 415, 236

Haisch, B. M., et al. 1981, ApJ, 245, 1009

Hall, D. S. 1976, in IAU Colloq. 29, Multiply Periodic Phenomena in

Variable Stars, ed. W. S. Fitch (Dordrecht: Reidel), 287

Havnes, O., \& Goertz, C. K. 1984, A\&A, 138, 421

Hjellming, R. M., Wade, C. M., \& Webster, E. 1972, Nature Phys. Sci. 246, 43

Holman, G. D. 1986, in Cool Stars, Stellar Systems, and the Sun, ed. M.

Zeilik \& D. M. Gibson (Berlin: Springer), 271

Hughes, V. A., \& McLean, B. J. 1984, ApJ, 278, 716

- 1987, ApJ, 313, 263

Jackson, P. D., Kundu, M. R., \& White, S. M. 1989, A\&A, 210, 284

Kahler, S., et al. 1982, ApJ, 252, 239

Katsova, M. M. 1987, in Activity in Cool Star Envelopes, ed. O. Havnes et al, (Dordrecht: Kluwer), 245

Kuijpers, J., \& van der Hulst, J. M. 1985, A\&A, 149, 343

Kundu, M. R. 1965, Solar Radio Astronomy (New York: Interscience)

Kundu, M. R., Jackson, P. D., White, S. M., \& Melozzi, M. 1987, ApJ, 312,822

Kundu, M. R., Pallavicini, R., White, S. M., \& Jackson, P. D. 1988, A\&A, 195,159

Kundu, M. R., \& Shevgaonkar, R. K. 1985, ApJ, 297, 644

Kundu, M. R., \& White, S. M. 1992, Mem. Soc. Astron. Ital., 63, 715

Lang, K. R., \& Willson, R. F. 1986, ApJ, 302, L17 1988a, ApJ, 326, 300 $1988 \mathrm{~b}, \mathrm{ApJ}, 328,610$

Leone, F., \& Umana, G. 1993, A\&A, 268, 667

Leous, J. A., Feigelson, E. D., André, P., \& Montmerle, T. 1991, ApJ, 379, 683

Lestrade, J.-F. 1988, in The Impact of VLBI on Astrophysics and Geophysics, ed. M. J. Reid \& J. M. Moran (Dordrecht: Kluwer), 265

Lestrade, J.-F., Mutel, R. L., Preston, R. A., \& Phillips, R. B. 1985, in Radio Stars, ed. R. M. Hjellming \& D. M. Gibson (Dordrecht: Reidel), 275

- 1986, in Cool Stars, Stellar Systems, and the Sun, ed. M. Zeilik \& D. M. Gibson (Berlin: Springer), 135

1988, ApJ, 328, 232

Lestrade, J.-F., Mutel, R. L., Preston, R. A., Scheid, J. A., \& Phillips, R. B. 1984, ApJ, 279, 184

Lim, J. 1993, ApJ, 405, L33

Lim, J., Nelson, G. J., Castro, C., Kilkenny, D., \& van Wyk, F. 1992, ApJ, 388, L27

Lin, R. P., Schwartz, R. A., Kane, S. R., Pelling, R. M., \& Hurley, K. C. 1984, ApJ, 283, 421
Linsky, J. L,, Drake, S. A., \& Bastian, T. S. 1992, ApJS, 393, 341

Linsky, J. L., \& Gary, D. E. 1983, ApJ, 274, 776

Massi, M., \& Chiuderi Drago, F. 1992, A\&A, 253, 403

Morris, D. H., \& Mutel, R. L. 1988, AJ, 95, 204

Morris, D. H., Mutel, R. L., \& Su, B. 1990, ApJ, 362, 299

Mullan, D. J. 1985, ApJ, 295, 628

Mullan, D. J., Doyle, J. G., Redman, R. O., \& Mathioudakis, M. 1992, ApJ, 397, 225

Mutel, R. L., Doiron, D. J., Lestrade, J.-F., \& Phillips, R. B. 1984, ApJ, 278,220

Mutel, R. L., \& Lestrade, J. F. 1985, AJ, 90, 493

Mutel, R. L., Lestrade, J.-F., Preston, R. A., \& Phillips, R. B. 1985, ApJ, 289,262

Mutel, R. L., Morris, D. H., Doiron, D. J., \& Lestrade, J.-F. 1987, AJ, 93, 1220

Mutel, R. L., \& Weisberg, J. M. 1978, AJ, 83, 1499

Owen, F. N., \& Gibson, D. M. 1978, AJ, 83, 1488

Owen, F. N., Jones, T. W., \& Gibson, D. M. 1976, ApJ, 210, L27

Owen, F. N., \& Spangler, S. R. 1977, ApJ, 217, L41

Pallavicini, R., Tagliaferri, G., \& Stella, L. 1990, A\&A, 228, 403

Pallavicini, R., Willson, R. F., \& Lang, K. R. 1985, A\&A, 149, 95

Phillips, R. B., \& Lestrade, J.-F. 1988, Nature, 334, 329

Phillips, R. B., Lonsdale, C. J., \& Feigelson, E. D. 1991, ApJ, 382, 261 1993, ApJ, 403, L43

Saar, S. H., \& Linsky, J. L. 1985, ApJ, 299, L47

Schmitt, J. H. M. M., Collura, A., Sciortino, S., Vaiana, G. S., Harnden, F. R., Jr., \& Rosner, R. 1990, ApJ, 365, 704

Schmitt, J. H. M. M., Pallavicini, R., Monsignori-Fossi, B. C., \& Harnden, F. R., Jr. 1987, A\&A, 179, 193

Skinner, S. L. 1993, ApJ, 408, 660

Skinner, S. L., \& Brown, A., 1994, in preparation

Skinner, S. L., Brown, A., \& Stewart, R. T. 1993, ApJS, 87, 217

Skumanich, A. 1985, Australian J. Phys., 38, 971

Slee, O. B., Nelson, G. J., Stewart, R. T., Wright, A. E., Innis, J. L., Ryan, S. G., Vaughan, A. E. 1987a, MNRAS, 229, 659

Slee, O. B., et al. 1987b, MNRAS, 227, 467

Slee, O. B., Stewart, R. T., Nelson, G. J., Wright, A. E., Dulk, G. A. Bastian, T. S., \& McKean, M. 1988, Astrophys. Lett., 27, 247

Spangler, S. R. 1977, AJ, 82, 169

Swank, J. H., \& Johnson, H. M. 1982, ApJ, 259, L67

Topka, K., \& Marsh, K. A. 1982, ApJ, 254, 641

Uchida, Y., \& Sakurai, T. 1983, in Activity in Red-Dwarf Stars, ed. P. B. Byrne \& M. Rodonò (Dordrecht: Reidel ), 629

Umana, G., Catalano, S., \& Rodonò, M. 1991, A\&A, 249, 217

Umana, G., Trigilio, C., Hjellming, R. M., Catalano, S., \& Rodonò, M. 1993, A\&A, 267, 126

Vaiana, G. S., et al. 1981, ApJ, 245, 163

van den Oord, G. H. J., Kuijpers, J., White, N. E., van der Hulst, J. M., \& Culhane, J. L. 1989, A\&A, 209, 296

Wade, C. M., \& Hjellming, R. M. 1972, Nature, 235, 270

Weiler, E. J., et al. 1978, ApJ, 225, 919

White, S. M., Jackson, P. D., \& Kundu, M. R. 1989a, ApJS, 71, 895

Whi $1993, \mathrm{AJ}, 105,563$

White, S. M., Kundu, M. R., \& Jackson, P. D. 1989b, A\&A, 225, 112

White, S. M., Kundu, M. R., Uchida, Y., \& Nitta, N. 1990, in Cool Stars,

Stellar Systems, and the Sun, ed. G. Wallerstein (San Francisco: ASP), 239

White, S. M., Pallavicini, R., \& Kundu, M. R. 1992a, A\&A, 257, 557 . 1992b, A\&A, 259, 149

Whitehouse, D. R. 1985, A\&A, 145, 449

Willson, R. F., \& Lang, K. R. 1987, ApJ, 312, 278

Willson, R. F., Lang, K. R., \& Foster, P. 1988, A\&A, 199, 255

Withbroe, G. L., \& Noyes, R. W. 1977, ARA\&A, 15, 363 